Original Research Article

\title{
Oral Dietary Supplements Use Among Healthcare Workers During the COVID-19 Pandemic in Malaysia
}

\author{
Heoy Teng Lee ${ }^{1}$, Hong Chuan Loh $^{1 *}$, Siti Nur Liyana Ramlee ${ }^{1}$, Irene Looi ${ }^{1,2}$ \\ Article History \\ Received: 9 August 2021; \\ Received in Revised Form: \\ 9 September 2021; \\ Accepted: 10 September \\ 2021; \\ Available Online: 20 \\ September 2021 \\ ${ }^{1}$ Clinical Research Centre, Hospital Seberang Jaya, Ministry of Health \\ Malaysia, Seberang Jaya 13700, Penang, Malaysia; leeheoyteng@gmail.com \\ (HTL); lohhongchuan@gmail.com (HCL); ctyana.ramlee@yahoo.com \\ (SNLR) \\ ${ }^{2}$ Medical Department, Hospital Seberang Jaya, Ministry of Health Malaysia, \\ Seberang Jaya 13700, Penang, Malaysia; irenelooi@yahoo.com (IL) \\ *Corresponding author: Hong Chuan Loh; Clinical Research Centre, Hospital \\ Seberang Jaya, Ministry of Health Malaysia, Seberang Jaya 13700, Penang, \\ Malaysia; lohhongchuan@gmail.com (HCL)
}

\begin{abstract}
Healthcare workers (HCWs) must be aware of the latest data supporting or refuting the use of oral dietary supplements (ODS) in order to disseminate evidence-based health information and help patients make informed decisions. Nevertheless, there is relatively scant data on the prevalence of dietary supplement use among HCWs, the types of dietary supplements recommended to patients by HCWs, and their reasons for recommending these products, particularly during the COVID-19 outbreak. This study examined the prevalence of ODS use among surveyed HCWs, considered the types of ODS used and recommended by those HCWs, identified the reasons given by those HCWs for using or not using ODS, and determined whether those HCWs were recommending or not recommending ODS to their patients during the COVID-19 pandemic. This cross-sectional study targeted all HCWs working at a district hospital in Malaysia and was conducted via a self-administered online survey. The survey revealed that $67.3 \%$ of HCWs did not recommend ODS for patient use during the pandemic, despite $55.3 \%$ of HCWs reported personal use of ODS during the pandemic. Type of HCWs $(P=0.001)$, monthly household income $(P=0.019)$, prior ODS use $(P<0.001)$ and recommendation of ODS to patients $(P<0.001)$ were significantly associated with personal ODS use during the pandemic. Vitamin $\mathrm{C}$ was the most commonly used $(81.3 \%)$ and recommended (95.0\%) ODS. "To maintain overall health and wellness" was the most common reason for personal ODS use and recommendation to patients $(83.3 \%$ \& $79.2 \%$ ). Given the increasing rate of ODS use during the pandemic, further research should be done so that evidence-based recommendations can be formulated to ensure patient safety.
\end{abstract}

Keywords: oral dietary supplements; healthcare workers; personal use; recommendation; COVID-19; pandemic

\section{Introduction}

Individuals exposed to risks and uncertainties tend to seek affordable self-protective measures such as complementary and alternative medicine (CAM), including taking oral 
dietary supplements (ODS), to maintain good health, mitigate risk, and make an unpredictable condition more manageable ${ }^{[1,2]}$. ODS are products that can be taken by mouth (such as a tablet, capsule, powder, or liquid), and include vitamins, minerals, herbs or other botanicals, amino acids, enzymes, tissues from organs or glands, or extracts of these. ODS are a common type of $\mathrm{CAM}^{[3,4]}$. Malaysia has undergone rapid urbanization in recent years resulting in the transformation of dietary intake patterns, including the consumption of dietary supplements. The most common ODS consumed by Malaysian adults was Vitamin $\mathrm{C}^{[3,5]}$.

Since the onset of the COVID-19 outbreak, some online media platforms have been promoting dietary supplements such as vitamins $\mathrm{C}$ and $\mathrm{D}$ for the treatment and prevention of coronavirus infection, although there is still a lack of evidence and guidance for the use of micronutrient supplements ${ }^{[6-8]}$. Recent scientific literature on the use of vitamins and minerals as prevention and treatment for critically ill patients with COVID-19 appear to support the idea of high-dose of vitamin $\mathrm{C}$ and other micronutrient intervention due to their excellent safety profile, low-cost and immediate availability ${ }^{[9-11]}$. The personal use of ODS by healthcare workers (HCWs) and the recommendation by HCWs for others to use ODS is of interest for several reasons. HCWs are important sources of dietary information for patients and are expected to provide sound advice about the use of various dietary supplements. Some HCWs provide information and dietary advice based on their personal experiences and eating habits. Therefore, we explored personal ODS use among HCWs themselves since their personal health habits could affect their decision to recommend a dietary supplement ${ }^{[12-14]}$. HCWs must be aware of the latest data supporting or refuting ODS use in order to disseminate evidence-based health information and help patients make informed decisions. Previous studies have shown that dietary supplements are used by a large proportion of the general public to treat and prevent diseases. Nevertheless, there is relatively scant data on the prevalence of dietary supplement use among HCWs. There is even less available up-to-date information regarding the types of dietary supplements recommended to patients by HCWs and their reasons for recommending these products, particularly during the COVID-19 outbreak ${ }^{[15-17]}$.

Therefore, this study was done to (a) examine the prevalence of ODS use among surveyed HCWs, (b) consider the types of ODS used and recommended by those HCWs, (c) identify the reasons given by those HCWs for using or not using ODS, and (d) determine whether those HCWs were recommending or not recommending ODS to their patients during the COVID-19 pandemic.

\section{Materials and Methods}

\subsection{Ethics Statement}

This study was registered with the Malaysian National Medical Research Register (NMRR-20-2275-56853) and received ethical approval from the Medical Research and Ethics Committee, Ministry of Health Malaysia. 


\subsection{Study Design and Participants}

This descriptive cross-sectional study was conducted via an anonymous, selfadministered online survey using a convenient sampling method. The targeted study population comprised all of the HCWs (including doctors, nurses, pharmacists, allied health professionals, and assistant medical officers) working at Hospital Seberang Jaya (HSJ), Malaysia. The time period for data collection was between $4^{\text {th }}$ November 2020 and $4^{\text {th }}$ December 2020. The survey was conducted using a self-administered online survey method in order to comply with the standard operating procedures imposed by the government during the COVID-19 pandemic. HCWs aged 18 years old and above were eligible to participate in the survey and were required to complete the electronic informed consent form. The study excluded non-Malaysian people and those who were unable to read or understand the questionnaire.

\subsection{Sample Size}

Cochran's formula was used to calculate the minimum recommended sampling size and an estimated proportion of dietary supplements users of 0.760 was calculated from a previous study ${ }^{[15]}$. The sample size required for this study with a confidence level of $95 \%$ and $\pm 5 \%$ precision was 280 study participants. A further $30 \%(N=84)$ was added to compensate for nonresponse, resulting in a final sample size of $365^{[18]}$.

\subsection{Survey Instrument}

Because previous research tools on ODS use among HCWs during the COVID-19 outbreak were unavailable, a semi-structured survey questionnaire instrument was developed for this study after doing a thorough literature review ${ }^{[3,16,17,19-25]}$ and seeking opinions from experts. To assess the content and face validation of the survey instrument, the questionnaire was reviewed by five researchers to evaluate appropriateness, relevancy, clarity, adequacy, and organization of the questions ${ }^{[26]}$. The questionnaire was pilot-tested to examine length, clarity, and difficulty of the questions, and items were revised based on the results. Responses obtained from the pilot study were not included in the final data analysis of this study. The study questionnaire was made available in the English language.

The finalised survey included 24 items with both multiple-choice and open-ended questions and consisted of four major sections. The participants were asked to complete their socio-demographic information, such as age, gender, marital status, ethnicity, religion, education level, type of HCWs, and monthly income, in the first section. The second section consisted of four questions on health-related characteristics. The third part had six questions about participants' personal ODS use before and during the COVID-19 pandemic $\left(11^{\text {th }}\right.$ March 2020 onwards); types of ODS consumed; reasons for taking or not taking ODS; and average expenditure per month for ODS. Available choices for types of ODS included vitamins $\mathrm{A}, \mathrm{B}$ complex, B12, C, D, and $\mathrm{E}$, as well as zinc, selenium, magnesium, multivitamin/multimineral (MVMM), omega-3 and probiotics, due to their potential utility 
in COVID-19 as had been highlighted in different studies ${ }^{[24,27-31]}$. Herbal products were excluded because they are not taught in traditional health care professional education programs. The fourth section contained five questions regarding the recommendation by HCW s that patients should or should not use ODS during the COVID-19 pandemic. The last section asked questions relating to participants' educational background regarding ODS and their interest in receiving continuing education regarding the subject of ODS.

\subsection{Statistical Analysis Methods}

The collected data of 367 participants was analysed statistically using the Statistical Package for Social Sciences (SPSS) version 20.0. Descriptive statistics were used to analyse the prevalence of ODS use and the socio-demographic and health-related characteristics of all HCWs involved in the study. Data on types of ODS used and recommended by HCWs to patients; reasons for using or not using ODS; and reasons for recommending or not recommending ODS to patients during the COVID-19 pandemic were summarized using frequency counts (n) and percentages (\%). Mean and standard deviation were calculated for the normally distributed continuous data whereas continuous data that were not normally distributed were reported using median and interquartile range (IQR). The ODS users and non-ODS users were compared by using the Mann-Whitney Test. Pearson Chi-square or Fisher's exact tests were performed to determine the categorical variables related to ODS use and any associations between ODS use and recommendation of ODS to patients. Tests were 2-tailed and P-value of less than 0.05 was considered statistically significant for all analyses.

\section{Results}

A total of $367 \mathrm{HCWs}$ participated in this study. Table 1 summarised the sociodemographic and health-related characteristics of all participants. The median age of participants was 32.00 years $(\mathrm{IQR}=9.00)$. Most of the participants were female $(87.2 \%)$, married (69.8\%), Malays (64.9\%) and Muslims (66.2\%). More than 90\% of the participants received tertiary education, did not smoke, and did not consume alcohol. Among the participants surveyed, $52.6 \%$ were nurses, $21 \%$ were pharmacists, $15.3 \%$ were doctors, and $11.2 \%$ were allied health professionals. Half of the participants $(51.0 \%)$ had monthly household income ranging from RM 4850 to RM 10 959. About 80\% of the participants had no underlying co-morbidities. A majority (99.5\%) of them were not infected with COVID19. However, there were 2 unconfirmed cases at the time the survey was conducted. The percentage of participants who had used ODS before the COVID-19 pandemic was $47.7 \%$ but during the pandemic $55.3 \%$ of participants used ODS. Despite that, the survey revealed that $67.3 \%$ of HCWs did not recommend ODS for patient use during the pandemic. Only 120 of the HCWs (32.7\%) recommended ODS to patients during the COVID-19 pandemic. 
Table 1. Sociodemographic and health characteristics of all participants $(n=367)$.

\begin{tabular}{|c|c|}
\hline Characteristics & $n(\%)$ \\
\hline Age $(\text { years })^{a}$ & $32(9.0)$ \\
\hline \multicolumn{2}{|c|}{ Gender } \\
\hline Female & $320(87.2)$ \\
\hline Male & $47(12.8)$ \\
\hline \multicolumn{2}{|c|}{ Marital status } \\
\hline Married & $256(69.8)$ \\
\hline Single & $104(28.3)$ \\
\hline Widowed & $4(1.1)$ \\
\hline Divorced & $3(0.8)$ \\
\hline \multicolumn{2}{|c|}{ Ethnicity } \\
\hline Malay & $238(64.9)$ \\
\hline Chinese & $78(21.3)$ \\
\hline Indian & $48(13.1)$ \\
\hline Others $^{\mathrm{b}}$ & $3(0.8)$ \\
\hline \multicolumn{2}{|c|}{ Religion } \\
\hline Islam & $243(66.2)$ \\
\hline Buddhism & $68(18.5)$ \\
\hline Hinduism & $40(10.9)$ \\
\hline Christianity & $16(4.4)$ \\
\hline \multicolumn{2}{|c|}{ Educational level } \\
\hline Secondary school & $14(3.8)$ \\
\hline Pre-University & $165(45.0)$ \\
\hline Undergraduate & $132(36.0)$ \\
\hline Postgraduate & $56(15.3)$ \\
\hline \multicolumn{2}{|c|}{ Type } \\
\hline Allied health professions & $41(11.2)$ \\
\hline Doctors & $56(15.3)$ \\
\hline Pharmacist & $77(21.0)$ \\
\hline Nurse & $193(52.6)$ \\
\hline \multicolumn{2}{|c|}{ Monthly household income } \\
\hline$<\mathrm{RM} 4850$ per month & $133(36.2)$ \\
\hline$\geq$ RM 4850 - RM 10959 per month & $187(51.0)$ \\
\hline > RM 10959 per month & $47(12.8)$ \\
\hline \multicolumn{2}{|c|}{ Smoking status } \\
\hline No & $359(97.8)$ \\
\hline Yes & $8(2.2)$ \\
\hline \multicolumn{2}{|c|}{ Alcohol consumption } \\
\hline No & $341(92.9)$ \\
\hline Yes & $26(7.1)$ \\
\hline \multicolumn{2}{|c|}{ Co-morbid(s) present } \\
\hline No & $295(80.4)$ \\
\hline Yes & $72(19.6)$ \\
\hline \multicolumn{2}{|c|}{ COVID-19 status } \\
\hline No & $365(99.5)$ \\
\hline Possibly infected & $2(0.5)$ \\
\hline Infected & $0(0.0)$ \\
\hline
\end{tabular}




\begin{tabular}{lc}
\hline Characteristics & \multicolumn{1}{c}{$\boldsymbol{n ( \% )}$} \\
\hline No & \multicolumn{1}{c}{ Prior ODS use } \\
Yes & $192(52.3)$ \\
& \multicolumn{1}{c}{ Use ODS during COVID-19 pandemic } \\
No & $164(44.7)$ \\
Yes & $203(55.3)$ \\
& Recommendation of ODS to patients \\
No & $247(67.3)$ \\
Yes & $120(32.7)$ \\
\hline
\end{tabular}

COVID-19 = Coronavirus disease 2019, ODS = Oral dietary supplements, RM = Ringgit Malaysia. ${ }^{\text {a }}$ Median (IQR), ${ }^{\mathrm{b}}$ Including Kadazan, Dusun, and Siamese

Table 2 summarizes all types of ODS used and recommended by HCWs to their patients. Among 367 participants, 203 (55.3\%) used ODS and 120 (32.7\%) recommended ODS to patients during the COVID-19 pandemic. It was found that the use of vitamin $\mathrm{C}$ was the highest $(81.3 \%)$, followed by Multivitamin/ multimineral (MVMM) supplement (17.7\%), vitamin B complex (17.7\%), omega-3 (10.3\%), and probiotics $(9.9 \%)$. Both vitamin C (95.0\%) and MVMM (32.5\%) were also the most common ODS recommended to patients during the pandemic, followed by omega-3 (12.5\%), vitamin B complex (10.0\%), and probiotics $(10.0 \%)$. HCWs were also asked how much on average they spent on ODS per month during the COVID-19 pandemic. The majority of the respondents (96.6\%) reported their monthly expenses on ODS in median (IQR) was RM100.00 (110.00).

Table 2. Types of ODS used and recommended by HCWs during the COVID-19 pandemic.

\begin{tabular}{ccccc}
\hline Types of ODS & \multicolumn{2}{c}{$\begin{array}{c}\text { Used personally } \\
(\boldsymbol{n = 2 0 3 )}\end{array}$} & $\begin{array}{c}\text { Recommended to patients } \\
(\boldsymbol{n = 1 2 0})\end{array}$ \\
\cline { 2 - 5 } & $\boldsymbol{n}$ & $\mathbf{\%}$ & $\boldsymbol{n}$ & $\boldsymbol{\%}$ \\
\hline Vitamin A & 6 & 3.0 & 3 & 2.5 \\
B complex vitamin & 36 & 17.7 & 12 & 10.0 \\
Vitamin B12 & 12 & 5.9 & 3 & 2.5 \\
Vitamin C & 165 & 81.3 & 114 & 95.0 \\
Vitamin D & 7 & 3.4 & 3 & 2.5 \\
Vitamin E & 18 & 8.9 & 6 & 5.0 \\
Zinc & 14 & 6.9 & 4 & 3.3 \\
Selenium & 1 & 0.5 & 0 & 0 \\
Magnesium & 6 & 3.0 & 1 & 0.8 \\
Multivitamin/Multimineral & 36 & 17.7 & 39 & 32.5 \\
Omega-3 & 21 & 10.3 & 15 & 12.5 \\
Probiotics & 20 & 9.9 & 12 & 10.0 \\
\hline
\end{tabular}

ODS = Oral dietary supplements, HCW = Healthcare workers, COVID-19 = Coronavirus disease 2019

Our survey questions on the reasons for using and recommending ODS during the COVID-19 pandemic are summarized in Table 3. The most frequently reported reason for taking ODS was "to maintain overall health and wellness" (83.3\%). The second highest reason was "to prevent getting infection" $(58.6 \%)$ followed by "to strengthen the immune system" (24.6\%). Similarly, the most commonly used reasons for recommending ODS to 
patients were "to maintain overall health and wellness" (79.2\%), "to prevent getting infection" (74.2\%), and "to strengthen the immune system" $(37.5 \%)$.

Table 3. Reasons for using and recommending ODS during the COVID-19 pandemic.

\begin{tabular}{ccccc}
\hline Reasons & \multicolumn{2}{c}{ Using ODS } \\
& \multicolumn{2}{c}{$\begin{array}{c}\text { Recommending ODS } \\
\text { n }\end{array}$} & \multicolumn{2}{c}{$\mathbf{n = 1 2 0}$} \\
\cline { 2 - 5 } & $\boldsymbol{n}$ & $\mathbf{\%}$ & $\boldsymbol{n}$ & $\mathbf{\%}$ \\
\hline To maintain overall health and wellness & 169 & 83.3 & 95 & 79.2 \\
Inadequate dietary intake and nutritional deficiency & 20 & 9.9 & 17 & 14.2 \\
To prevent getting an infection & 50 & 24.6 & 45 & 37.5 \\
To strengthen the immune system & 119 & 58.6 & 89 & 74.2 \\
To help cope with the adverse effects of conventional treatment & 3 & 1.5 & 4 & 3.3 \\
To help in the treatment of a specific disease & 12 & 5.9 & 8 & 6.7 \\
Conventional treatment/modern medicine is not effective & 1 & 0.5 & 0 & 0.0 \\
\hline
\end{tabular}

ODS = Oral dietary supplements, COVID-19 = Coronavirus disease 2019.

Meanwhile, Table 4 presented the feedback of the HCWs on the reasons for not using and not recommending ODS during the COVID-19 pandemic. Nearly $44 \%$ of them reported "dietary intake is adequate" as the main reason for not using ODS. Other reasons for not using ODS included "worried about its safety and adverse effects" (31.1\%) which was second, followed by "do not know much about dietary supplements" (17.1\%) and "worried about its potential drug-dietary supplement interactions" (11.6\%). In contrast, the most common reasons for not recommending ODS to patients were "worried about its safety and adverse effects" (34.8\%) and "worried about its potential drug-dietary supplement interactions" (31.6\%), followed by "lack of scientific evidence on its effectiveness in disease treatment" (23.5\%), "dietary intake is adequate" $(22.3 \%)$, and "do not know much about dietary supplements" (18.6\%).

Table 4. Reasons for not using and not recommending ODS during the COVID-19 pandemic.

\begin{tabular}{|c|c|c|c|c|}
\hline \multirow[t]{2}{*}{ Reasons } & \multicolumn{2}{|c|}{$\begin{array}{c}\text { Not using ODS } \\
\quad n=164 \\
\end{array}$} & \multicolumn{2}{|c|}{$\begin{array}{l}\text { Not recommending ODS } \\
\qquad n=247\end{array}$} \\
\hline & $n$ & $\%$ & $n$ & $\%$ \\
\hline $\begin{array}{c}\text { Lack of scientific evidence on its effectiveness in disease } \\
\text { treatment }\end{array}$ & 13 & 7.9 & 58 & 23.5 \\
\hline $\begin{array}{l}\text { Worried about its potential drug-dietary supplement } \\
\text { interactions }\end{array}$ & 19 & 11.6 & 78 & 31.6 \\
\hline Worried about its safety and adverse effects & 51 & 31.1 & 86 & 34.8 \\
\hline Dietary supplements are expensive & 11 & 6.7 & 28 & 11.3 \\
\hline Do not know much about dietary supplements & 28 & 17.1 & 46 & 18.6 \\
\hline Satisfied with conventional treatment/modern medicine & 17 & 10.4 & 18 & 7.3 \\
\hline Dietary intake is adequate & 72 & 43.9 & 55 & 22.3 \\
\hline
\end{tabular}

ODS = Oral dietary supplements, COVID-19 = Coronavirus disease 2019.

The association among socio-demographic, health-related characteristics, and ODS use during the COVID-19 pandemic is depicted in Table 5. We observed that personal ODS use during the COVID-19 pandemic was not associated with age, gender, marital status, ethnicity, religion, educational level, smoking status, alcohol consumption, or underlying non-communicable diseases. However, type of HCWs $(P=0.001)$, monthly household 
income $(P=0.019)$, prior ODS use $(P<0.001)$, and recommendation of ODS to patients $(P<0.001)$ were significantly associated with personal ODS use during the COVID-19 pandemic, tested using Pearson Chi-square test.

Table 5. Association between socio-demographic, health-related characteristics, and ODS use during the COVID-19 pandemic.

\begin{tabular}{|c|c|c|c|c|}
\hline Characteristics & $n$ & $\begin{array}{c}\text { ODS users } \\
n=203 \\
n(\%)\end{array}$ & $\begin{array}{c}\text { Non-users } \\
n=164 \\
n(\%)\end{array}$ & $P$-value \\
\hline Age in years, median (IQR) & 367 & $32.0(8.0)$ & $31.5(11.0)$ & $0.811^{\mathrm{a}}$ \\
\hline Gender & & & & $0.060^{\mathrm{b}}$ \\
\hline Female & 320 & $183(90.1)$ & $137(83.5)$ & \\
\hline Male & 47 & $20(9.9)$ & $27(16.5)$ & \\
\hline Marital status & & & & $0.471^{\mathrm{c}}$ \\
\hline Single & 104 & $55(27.1)$ & $49(29.9)$ & \\
\hline Married & 256 & $146(71.9)$ & $110(67.1)$ & \\
\hline Widowed & 4 & $1(0.5)$ & $3(1.8)$ & \\
\hline Divorced & 3 & $1(0.5)$ & $2(1.2)$ & \\
\hline Ethnicity & & & & $0.662^{c}$ \\
\hline Malay & 238 & $128(63.1)$ & $110(67.1)$ & \\
\hline Chinese & 78 & $47(23.2)$ & $31(18.3)$ & \\
\hline Indian & 48 & $27(13.3)$ & $21(12.8)$ & \\
\hline Others $^{\mathrm{d}}$ & 3 & $1(0.5)$ & $2(1.2)$ & \\
\hline Religion & & & & $0.164^{\mathrm{b}}$ \\
\hline Islam & 243 & $129(63.5)$ & $114(69.5)$ & \\
\hline Buddhism & 68 & $43(21.2)$ & $25(15.2)$ & \\
\hline Christianity & 16 & $6(3.0)$ & $10(6.1)$ & \\
\hline Hinduism & 40 & $15(7.4)$ & $25(15.2)$ & \\
\hline Educational level & & & & $0.232^{\mathrm{b}}$ \\
\hline Secondary school & 14 & $5(2.5)$ & $9(5.5)$ & \\
\hline Pre-University & 165 & $88(43.3)$ & $77(47.0)$ & \\
\hline Undergraduate & 132 & $74(36.5)$ & $58(35.4)$ & \\
\hline Postgraduate & 56 & $36(17.7)$ & $20(12.2)$ & \\
\hline Type & & & & $0.001^{\mathrm{b}}$ \\
\hline Allied health professions & 41 & $12(6.0)$ & $29(17.7)$ & \\
\hline Doctors & 56 & $28(13.8)$ & $28(17.1)$ & \\
\hline Pharmacist & 77 & $51(25.1)$ & $26(15.9)$ & \\
\hline Nurse & 193 & $112(55.2)$ & $81(49.4)$ & \\
\hline Monthly household income & & & & $0.019^{\mathrm{b}}$ \\
\hline$<$ RM 4850 per month & 133 & $63(31.0)$ & $70(42.7)$ & \\
\hline$\geq$ RM 4850 - RM 10959 per month & 187 & $107(52.7)$ & $80(48.8)$ & \\
\hline$>$ RM 10959 per month & 47 & $33(16.3)$ & $14(8.5)$ & \\
\hline Smoking status & & & & $0.736^{\mathrm{c}}$ \\
\hline No & 359 & $198(97.5)$ & $161(98.2)$ & \\
\hline Yes & 8 & $5(2.5)$ & $3(1.8)$ & \\
\hline Alcohol consumption & & & & $0.284^{\mathrm{b}}$ \\
\hline No & 341 & $186(91.6)$ & $155(94.5)$ & \\
\hline Yes & 26 & $17(8.4)$ & $9(5.5)$ & \\
\hline Co-morbid(s) present & & & & $0.827^{\mathrm{b}}$ \\
\hline No & 295 & $164(80.8)$ & $131(79.9)$ & \\
\hline Yes & 72 & $39(19.2)$ & $33(20.1)$ & \\
\hline
\end{tabular}




\begin{tabular}{ccccc}
\hline Characteristics & $\boldsymbol{n}$ & $\begin{array}{c}\text { ODS users } \\
\boldsymbol{n}=\mathbf{2 0 3} \\
\boldsymbol{n}(\boldsymbol{\%})\end{array}$ & $\begin{array}{c}\text { Non-users } \\
\boldsymbol{n}=\mathbf{1 6 4} \\
\boldsymbol{n}(\boldsymbol{\%})\end{array}$ & P-value \\
\hline Prior ODS use & 192 & $31(15.3)$ & $161(98.2)$ & \\
No & 175 & $172(84.7)$ & $3(1.8)$ & \\
Yes & & & & $<0.001^{\mathrm{b}}$ \\
Recommendation of ODS to patients & 247 & $104(51.2)$ & $143(87.2)$ & \\
No & 120 & $99(48.8)$ & $21(12.8)$ & \\
\hline
\end{tabular}

COVID-19 = Coronavirus disease 2019, ODS = Oral dietary supplements, RM = Ringgit Malaysia. ${ }^{\text {a }}$ MannWhitney Test, ${ }^{\mathrm{b}}$ Pearson Chi-square test, ${ }^{\mathrm{c}}$ Fisher's exact test, ${ }^{\mathrm{d}}$ Including Kadazan, Dusun, and Siamese.

Table 6 shows that most of the HCWs involved in this study did not receive any formal education or training about ODS $(77.9 \%)$ but were interested in receiving continuing education about ODS (86.9\%).

Table 6. HCWs' education background and interest in receiving continuing education about ODS $(n=367)$.

\begin{tabular}{ccc}
\hline Variables & $\boldsymbol{n}$ & $\mathbf{( \% )}$ \\
\hline Received formal education or training about ODS & 286 & $(77.9)$ \\
No & 81 & $(2.1)$ \\
Yes & & \\
Interested in receiving continuing education about ODS & 48 & $(13.1)$ \\
No & 319 & $(86.9)$ \\
\hline
\end{tabular}

HCW $=$ Healthcare workers, ODS $=$ Oral dietary supplements, TV $=$ Television. ${ }^{a}$ Including Google Search and Nurse.

\section{Discussion}

To our knowledge, this study is the first in the country to examine the use of ODS among HCWs during the COVID-19 pandemic. Socio-demographic and health-related characteristics were explored to understand the association of these factors with ODS use among HCWs. As such, the findings of this study will hopefully help support the development of nutritional guidelines and policies, education programs, and future studies related to dietary supplements in order to guide HCWs in providing the best advice to patients.

According to our survey, almost half of HCWs had used ODS before the COVID-19 pandemic. The Malaysian Adults Nutrition Survey 2014 (MANS 2014) conducted during a non-pandemic period among Malaysian adults showed that the prevalence of ODS use among adults in the general population was $59.1 \%{ }^{[3]}$. Notably, the data showed that the use of ODS was less common among HCWs as compared to the general population during the nonpandemic period. Nevertheless, the consumption of ODS among HCWs increased during the COVID-19 pandemic as indicated by our study. The recent PLifeCOVID-19 Online Study conducted in Poland revealed that ODS was consumed more often during the first wave of the pandemic than in the second ${ }^{[32]}$. This trend might be due to the belief that ODS can give benefits and potentially prevent and help manage a variety of health conditions, including COVID-19 and other infections, although evidence to prove their efficacy with COVID-19 
is still lacking ${ }^{[8,24,27,31,33-35]}$. Similarly, a study in Korea also demonstrated an overall rise in CAM use by $21.4 \%$ during the Middle East Respiratory Syndrome outbreak among outpatients $^{[16]}$. Another study also found that dietary supplements were one of the most commonly used CAM by patients with dengue fever ${ }^{[21]}$. Therefore, like other members of the public, HCWs could be expected to consume dietary supplements for their health benefits during the outbreak of disease.

Our results suggest that the type of HCW (e.g., doctor, nurse, etc.) influenced the personal use of ODS during the COVID-19 pandemic. Concerning each type of HCW, ODS use was higher among nurses $(55.2 \%)$, followed by pharmacists $(25.1 \%)$, doctors $(13.8 \%)$ and allied health professionals $(6.0 \%)$. For comparison, the Healthcare Professionals Impact Study found that $72 \%$ of physicians and $89 \%$ of nurses used dietary supplements regularly, occasionally, or seasonally, whereas $51 \%$ of physicians and $59 \%$ of nurses used dietary supplements regularly ${ }^{[15]}$. It is challenging to obtain reliable estimates of the prevalence of ODS use due to differences in definitions, frequency of use, diversity of dietary supplement formulations, and availability of dietary supplements ${ }^{[5,36]}$. Overall, previous studies showed the prevalence of ODS use varying among HCWs, ranging from $21 \%$ to $88 \%^{[12,37-39]}$. This study also found that monthly household income was significantly associated with the use of ODS. Those with a monthly income of more than RM 4850 showed higher ODS use than those with a monthly income of less than RM 4 850. Interestingly, those with a monthly income of more than RM 10959 showed the least use of ODS despite having a higher income. Nevertheless, our findings were consistent with previous studies suggesting that the relationship between ODS use among HCWs and monthly income remains unclear ${ }^{[3,40]}$.

Apart from that, our study revealed a positive association between prior ODS use, recommendation of ODS to patients, and the use of ODS during the COVID-19 pandemic. Among ODS users during the pandemic, most of them had used ODS before the pandemic. Our study found that the majority of HCWs did not recommend ODS to patients. However, among those who did recommend ODS to patients, a large proportion of them used ODS during the COVID-19 pandemic themselves, which result was similar to other studies ${ }^{[17,41,42]}$. One previous study revealed that personal use of dietary supplements was associated with a twofold increase in the likelihood that a pharmacist would recommend a dietary supplement to others ${ }^{[43]}$.

Our results showed that the most commonly used and recommended ODS during the pandemic was vitamin C, followed by MVMM, and these results concurred with previous studies conducted in Malaysia and other countries before the COVID-19 outbreak ${ }^{[3,15,16,38,44]}$. Another recent study conducted in Saudi Arabia during the COVID-19 pandemic discovered that almost all of the ODS users reported taking vitamin $\mathrm{C}^{[45]}$. The high use of vitamin $\mathrm{C}$ could be related to recent studies that promoted vitamin C's potential benefits as a prophylactic intervention to combat viral infection and as an immune modulator to mitigate the risk of contracting severe COVID-19 ${ }^{[10,46-49]}$. Selenium appeared to be the least consumed and recommended among HCWs which could be due to limited knowledge about this 
supplement among $\mathrm{HCWs}^{[50]}$. Nevertheless, previous studies suggest that it could contribute to the prevention and management of COVID-19 and other viral infections ${ }^{[50,51]}$.

Our survey also revealed that HCWs commonly used ODS to maintain their overall health and wellness, strengthen their immune system, and prevent getting an infection during the COVID-19 pandemic. These findings were similar to the reasons reported in previous surveys of other HCWs and the general population during the non-pandemic period ${ }^{[15,17,52,53]}$. Most of them used ODS as a complementary therapy and a primary prevention intervention rather than a substitute for conventional treatment. ODS should not replace a healthy and balanced diet. Promoting the health effects of ODS in a generally healthy population and, in particular, their effectiveness during a pandemic warrant further investigation ${ }^{[34,54,55]}{ }^{\text {. Since }}$ ODS, especially vitamin C and MVMM, are thought to boost immunity against infection during the pandemic, more studies or clinical trials should be conducted to explore their possible beneficial effects in prevention or management of disease. It is crucial to explore the exact intracellular mechanisms and anti-inflammatory and anti-oxidative activities of $\mathrm{ODS}^{[49,56]}$.

While the majority of participants in this study used ODS during the pandemic, there were still almost half of them $(44.7 \%)$ who did not. Most of those who did not use ODS believed that their dietary intake is adequate. Therefore, dietary guidelines provided by governing bodies play a crucial role in ensuring proper nutritional practices, such as eating a balanced diet and maintaining optimal health ${ }^{[57]}$. Most of the HCWs who did not recommend the use of ODS to patients had concerns about their safety and possible adverse effects as well as potential drug-dietary supplement interactions. Indeed, many marketed ODS or nutraceutical products are not strictly regulated, thus their safety and efficacy can be questioned $^{[58]}$. It is important to evaluate the evidence regarding the use of such products especially relating to their safety, adverse effects, and drug interactions. Data from ongoing large randomized trials will be necessary to establish the role of ODS during a disease outbreak or pandemic.

In addition, our survey also revealed that most of the HCWs had not received any formal education or training regarding ODS but were interested to learn about it. In agreement with prior studies, most of the HCWs who were likely to recommend that their patients consume ODS would be interested in continuing medical education courses and training on various ODS therapies ${ }^{[59,60]}$. The majority of HCWs expressed a marked preference and interest in integrative medicine modalities in the management of certain types of infectious diseases and their complications ${ }^{[60]}$. Accordingly, it is hoped that our findings will help government, policymakers, and international organizations in efforts to provide effective guidelines and evidence-based recommendations for patient safety.

\section{Limitation}

Several limitations were encountered throughout this study. This was a questionnairebased cross-sectional study in which we relied completely on information provided by the participants, and their responses could have been biased or based on a misunderstanding of 
the questions. The results obtained are based on self-reported data rather than on direct observation or by interview which could over or underestimate the true use of ODS. Our study was conducted at only one district hospital and it is possible that the results may not be generalized to the majority of HCW in our country. In addition, the survey sample was not selected with true randomization methods but rather relied on a convenience sample. These limitations may affect the generalizability of the results. Nevertheless, it provides useful information and may serve as a benchmark for further study.

\section{Conclusion}

In summary, further research is required before evidence-based recommendations can be formulated. HCWs still share responsibility for providing correct information about ODS use. Given the weak evidence relating to the use of many ODS, there is a need to strengthen the health science curriculum to produce better informed future professionals. Lastly, the use of ODS should be evidence-based to ensure patient safety.

Author Contributions: Conceptualization by HTL, HCL; methodology prepared by HTL, HCL; validation by HTL, HCL, IL; formal analysis by HTL, HCL; investigation by HTL, HCL; resources by HTL, HCL, IL; writing - original draft preparation by HTL; writing - review and editing by HCL, SNLR, and IL; supervision by HCL, IL; project administration by HTL, HCL, SNLR. All authors read and approved the final manuscript.

Funding: No external funding was provided for this research.

Acknowledgments: The authors would like to honour all the HCWs around the world who dedicated themselves bravely and tirelessly to patient care in the fight against COVID-19. Also, the authors would like to express gratitude to all the participants for their cooperation in completing the survey questionnaire, all of whom directly and indirectly assisted in the success of this study. We thank the Director-General of Health Malaysia for permission to present these findings.

Conflicts of Interest: The authors declare no conflict of interest.

\section{References}

1. Mitchell, M, McClean, S. Pregnancy, risk perception and use of complementary and alternative medicine. Health Risk Soc 2014; 16(1): 101-116.

2. Omar, UH, Putit, L. Consumer behavioral intention to use complementary alternative medicine. Identity 2012; 3: H5.

3. Zaki, A, Rasidi, N, Awaluddin, SM, et al., Prevalence and characteristic of dietary supplement users in Malaysia: data from the Malaysian Adult Nutrition Survey (MANS) 2014. Glob J Health Sci 2018; 10: 127 .

4. Marx, W, Kiss, N, McKavanagh, D, et al., Attitudes, beliefs and behaviours of Australia dietitians regarding dietary supplements: A cross-sectional survey. Complement Ther Clin Pract 2016; 25: 8791.

5. National Institutes of Health. Multivitamin/mineral Supplements Fact Sheet for Health Professionals. 2019 [Accessed 2020 October 17]; Available from: https://ods.od.nih.gov/factsheets/MVMSHealthProfessional/.

6. Islam, MS, Sarkar, T, Khan, SH, et al., COVID-19-related infodemic and its impact on public health: a global social media analysis. Am J Trop Med Hyg 2020; 103(4): 1621-1629. 
7. Anwar, A, Malik, M, Raees, V, et al., Role of mass media and public health communications in the COVID-19 pandemic. Cureus 2020; 12(9): e10453.

8. Lanham-New, SA, Webb, AR, Cashman, KD, et al., Vitamin D and SARS-CoV-2 virus/COVID-19 disease. BMJ Nutr Prev Health 2020; 3(1): 106-110.

9. Bae, M, Kim, H, Mini-review on the roles of vitamin C, vitamin D, and selenium in the immune system against COVID-19. Molecules 2020; 25(22): 5346.

10. Carr, AC, Rowe, S, The emerging role of vitamin C in the prevention and treatment of COVID-19. Nutrients 2020; 12(11).

11. Abobaker, A, Alzwi, A, Alraied, AHA, Overview of the possible role of vitamin C in management of COVID-19. Pharmacol Rep 2020; 72(6): 1517-1528.

12. Frank, E, Bendich, A, Denniston, M, Use of vitamin-mineral supplements by female physicians in the United States. Am J Clin Nutr 2000; 72(4): 969-975.

13. Frank, E, Kunovichfrieze, T. Physicians' prevention counseling behaviors: current status and future directions. Prev Med 1995; 24(6): 543-545.

14. Institute of Medicine (US) Committee on Dietary Guidelines Implementation. Health-care professionals: strategies and actions for implementation. In: Improving America's Diet and Health: From Recommendations to Action.PR Thomas, Washington (DC): National Academies Press (US); 1991: 168-183.

15. Dickinson, A, Boyon, N, Shao, A, Physicians and nurses use and recommend dietary supplements: report of a survey. Nutr J 2009; 8(1): 29.

16. Hwang, JH, Cho, HJ, Im, HB, et al., Complementary and alternative medicine use among outpatients during the 2015 MERS outbreak in South Korea: a cross-sectional study. BMC Complement Med Ther 2020; 20(1).

17. Marupuru, S, Axon, DR, Slack, MK, How do pharmacists use and recommend vitamins, minerals, herbals and other dietary supplements? BMC Complement Altern Med 2019; 19(1).

18. Israel, G, Determining sample size. Florida Cooperative Extension Service, Institute of Food and Agricultural Sciences, University of Florida 1992 1992: PEOD6:1-5.

19. Abd Wahab, MS, Abd Malik, NA, Sahudin, S, et al., Exploring the factors associated with the intention to assess customers' herbal and dietary supplement use by community pharmacists in Kuala Lumpur, Malaysia. J Appl Pharm Sci 2019; 9(12): 108-116.

20. Kemper, KJ, Gardiner, P, Gobble, J, et al., Expertise about herbs and dietary supplements among diverse health professionals. BMC Complement Altern Med 2006; 6(1).

21. Ching, S, Ramachandran, V, Gew, LT, et al., Complementary alternative medicine use among patients with dengue fever in the hospital setting: a cross-sectional study in Malaysia. BMC Complement Altern Med 2016; 16(1): 37.

22. Tangkiatkumjai, M, Boardman, H, Praditpornsilpa, K, et al., Prevalence of herbal and dietary supplement usage in Thai outpatients with chronic kidney disease: a cross-sectional survey. BMC Complement Altern Med 2013; 13(1): 153.

23. Derwand, $\mathrm{R}$ and Scholz, $\mathrm{M}$, Does zinc supplementation enhance the clinical efficacy of chloroquine/hydroxychloroquine to win today's battle against COVID-19? Med Hypotheses 2020; 142: 109815. 
24. Jovic, TH, Ali, SR, Ibrahim, N, et al., Could vitamins help in the fight against COVID-19? Nutrients 2020; 12(9).

25. Portella, CFS, Ghelman, R, Abdala, CVM, et al., Evidence map on the contributions of traditional, complementary and integrative medicines for health care in times of COVID-19. Integr Med Res 2020; 9(3): 100473.

26. Taherdoost, H, Validity and reliability of the research instrument; how to test the validation of a questionnaire/qurvey in a research. SSRN Electronic Journal 2016.

27. Alexander, J, Tinkov, A, Strand, TA, et al., Early nutritional interventions with zinc, selenium and vitamin D for raising anti-viral resistance against progressive COVID-19. Nutrients 2020; 12(8).

28. Jayawardena, R, Sooriyaarachchi, P, Chourdakis, M, et al., Enhancing immunity in viral infections, with special emphasis on COVID-19: A review. Diabetes Metab Syndr: Clin Res Rev 2020; 14(4): 367-382.

29. Orrù, B, Szekeres-Bartho, J, Bizzarri, M, et al., Inhibitory effects of Vitamin D on inflammation and IL-6 release. A further support for COVID-19 management? Eur Rev Med Pharmacol Sci 2020; 24(15): 8187-8193.

30. Wu, D, Lewis, ED, Pae, M, et al., Nutritional modulation of immune function: analysis of evidence, mechanisms, and clinical relevance. Front Immunol 2019; 9(3160).

31. Mousa, HA-L, Prevention and treatment of influenza, influenza-like illness, and common cold by herbal, complementary, and natural therapies. J Evid Based Complementary Altern Med 2017; 22(1): $166-174$.

32. Hamulka, J, Jeruszka-Bielak, M, Górnicka, M, et al., Dietary supplements during COVID-19 outbreak. Results of Google trends analysis supported by PLifeCOVID-19 online studies. Nutrients 2020; 13(1): 54.

33. Singh, V, Can vitamins, as epigenetic modifiers, enhance immunity in COVID-19 patients with noncommunicable disease? Curr Nutr Rep 2020; 9(3): 202-209.

34. Adams, KK, Baker, WL, Sobieraj, DM, Myth busters: dietary supplements and COVID-19. Ann Pharmacother 2020; 54(8): 820-826.

35. Ali, N, Role of vitamin D in preventing of COVID-19 infection, progression and severity. J Infect Public Health 2020; 13(10): 1373-1380.

36. Dwyer, JT, Coates, PM, Smith, MJ, Dietary supplements: regulatory challenges and research resources. Nutrients 2018; 10(1): 41.

37. Spencer, EH, Bendich, A, Frank, E, Vitamin and mineral supplement use among US Medical students: a longitudinal study. J Am Diet Assoc 2006; 106(12): 1975-1983.

38. Kemper, KJ, Gardiner, P, Woods, C, Changes in use of herbs and dietary supplements (HDS) among clinicians enrolled in an online curriculum. BMC Complement Altern Med 2007; 7(1): 21.

39. Box, S, Creswell, B, Hagan, DW, Alternative health care education in dietetic training programs: a survey of perceived needs. J Am Diet Assoc 2001; 101(1): 108-110.

40. Cowan, AE, Jun, S, Gahche, JJ, et al., Dietary supplement use differs by socioeconomic and healthrelated characteristics among U.S. Adults, NHANES 2011²014. Nutrients 2018; 10(8): 1114.

41. Cashman, LS, Burns, JT, Otieno, IM, et al., Massachusetts registered dietitians' knowledge, attitudes, opinions, personal use, and recommendations to clients about herbal supplements. J Altern Complement Med 2003; 9(5): 735-46. 
42. Welna, EM, Hadsall, RS, Schommer, JC, Pharmacists' personal use, professional practice behaviors, and perceptions regarding herbal and other natural products. J Am Pharm Assoc 2003; 43(5): 602-11.

43. Howard, N, Tsourounis, C, Kapusnik-Uner, J, Dietary supplement survey of pharmacists: personal and professional practices. J Altern Complement Med 2001; 7(6): 667-680.

44. Gardiner, P, Woods, C, Kemper, KJ, Dietary supplement use among health care professionals enrolled in an online curriculum on herbs and dietary supplements. BMC Complement Altern Med 2006; 6: 21.

45. Alyami, HS, Orabi, MAA, Aldhabbah, FM, et al., Knowledge about COVID-19 and beliefs about and use of herbal products during the COVID-19 pandemic: A cross-sectional study in Saudi Arabia. Saudi Pharm J 2020; 28(11): 1326-1332.

46. Colunga Biancatelli, RML, Berrill, M, Catravas, JD, et al., Quercetin and vitamin C: an experimental, synergistic therapy for the prevention and treatment of SARS-CoV-2 related disease (COVID-19). Front Immunol 2020; 11: 1451-1451.

47. Gasmi, A, Noor, S, Tippairote, T, et al., Individual risk management strategy and potential therapeutic options for the COVID-19 pandemic. Clin Immunol 2020; 215: 108409.

48. Liu, F, Zhu, Y, Zhang, J, et al., Intravenous high-dose vitamin C for the treatment of severe COVID19: study protocol for a multicentre randomised controlled trial. BMJ Open 2020; 10(7): e039519e039519.

49. Cheng, RZ, Can early and high intravenous dose of vitamin C prevent and treat coronavirus disease 2019 (COVID-19)? Medicine in Drug Discovery 2020; 5: 100028.

50. Kieliszek, M, Lipinski, B, Selenium supplementation in the prevention of coronavirus infections (COVID-19). Med Hypotheses 2020; 143: 109878-109878.

51. Guillin, OM, Vindry, C, Ohlmann, T, et al., Selenium, selenoproteins and viral infection. Nutrients 2019; 11(9).

52. Islahudin, F, Shahdan, IA, and Mohamad-Samuri, S, Association between belief and attitude toward preference of complementary alternative medicine use. Patient Prefer Adherence 2017; 11: 913-918.

53. Bailey, RL, Gahche, JJ, Miller, PE, et al., Why US adults use dietary supplements. JAMA Intern Med 2013; 173(5): 355-361.

54. Zhang, FF, Barr, SI, McNulty, H, et al., Health effects of vitamin and mineral supplements. BMJ (Clinical research ed) 2020; 369: $\mathrm{m} 2511-\mathrm{m} 2511$.

55. Lordan, R, Rando, HM, Consortium, C-R, et al., Dietary supplements and nutraceuticals under investigation for COVID-19 prevention and treatment. ArXiv 2021: arXiv:2102.02250v1.

56. Alagawany, M, Attia, YA, Farag, MR, et al., The strategy of boosting the immune system under the COVID-19 pandemic. Front Vet Sci 2021; 7(712).

57. World Health Organization, Food and nutrition tips during self-quarantine. 2020, Organization Geneva.

58. Domínguez Díaz, L, Fernández-Ruiz, V, and Cámara, M, The frontier between nutrition and pharma: the international regulatory framework of functional foods, food supplements and nutraceuticals. Crit Rev Food Sci Nutr 2020; 60(10): 1738-1746.

59. Stussman, BJ, Nahin, RR, Barnes, PM, et al., U.S. physician recommendations to their patients about the use of complementary health approaches. J Altern Complement Med 2020; 26(1): 25-33. 
60. Shere-Wolfe, KD, Tilburt, JC, D'Adamo, C, et al., Infectious diseases physicians' attitudes and practices related to complementary and integrative medicine: results of a national survey. Evid Based Complement Alternat Med 2013; 2013: 294381.

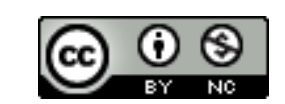

Author(s) shall retain the copyright of their work and grant the Journal/Publisher right for the first publication with the work simultaneously licensed under:

Creative Commons Attribution-NonCommercial 4.0 International (CC BY-NC 4.0). This license allows for the copying, distribution and transmission of the work, provided the correct attribution of the original creator is stated. Adaptation and remixing are also permitted. 